\title{
Efficient induction of antimicrobial activity with vancomycin nanoparticle-loaded poly(trimethylene carbonate) localized drug delivery system
}

This article was published in the following Dove Press journal:

International Journal of Nanomedicine

10 February 2017

Number of times this article has been viewed

\author{
Yang Zhang ${ }^{1, *}$ \\ Ruo-jia Liang ${ }^{2, *}$ \\ Jiao-jiao $\mathrm{Xu}^{3}$ \\ Li-feng Shen' \\ Jian-qing $\mathrm{GaO}^{4}$ \\ Xu-ping Wang' \\ Na-ni Wang' \\ Dan Shou' \\ Ying $\mathrm{Hu}^{3}$
}

'Department of Medicine, Zhejiang Academy of Traditional Chinese Medicine, ${ }^{2}$ Department of Gynaecology, Zhejiang Provincial Hospital of Traditional Chinese Medicine, Hangzhou, ${ }^{3}$ Zhejiang Pharmaceutical College, Ningbo, ${ }^{4}$ College of Pharmaceutical Sciences, Zhejiang University, Hangzhou, People's Republic of China

*These authors contributed equally to this work

\begin{abstract}
Surgery and the local placement of an antibiotic are the predominant therapies to treat chronic osteomyelitis. Vancomycin-loaded $N$-trimethyl chitosan nanoparticles (VCM/ TMC NPs) as a potential drug delivery system have high intracellular penetration and effective intracellular antibacterial activity. This study investigated the effects of a biocompatible material, poly(trimethylene carbonate) (PTMC), to increase the sustained effectiveness of an intracellular antibiotic and its potential application in antibiotic delivery. VCM/TMC NP-PTMC was characterized using scanning electron microscopy and Fourier transform infrared spectroscopy to determine the morphology, stability and chemical interaction of the drug with the polymer. Further, the biodegradation, antibacterial activity, protein adsorption, cell proliferation and drug release characteristics were evaluated. In addition, a Staphylococcus aureus-induced osteomyelitis rabbit model was used to investigate the antibiotic activity and bone repair capability of VCM/TMC NP-PTMC. The results showed that the composite beads of VCM/TMC NPs followed a sustained and slow release pattern and had excellent antibacterial activity and a higher protein adsorption and cell proliferation rate than the VCM-PTMC in vitro. Furthermore, VCM/TMC NP-PTMC inhibits bacteria and promotes bone repair in vivo. Thus, VCM/TMC NP-PTMC might be beneficial in periodontal management to reduce the bacterial load at the infection site and promote bone repair.
\end{abstract}

Keywords: vancomycin-loaded $N$-trimethyl chitosan nanoparticles, poly(trimethylene carbonate), sustained release, intracellular antibiotic effect, bone infection

\section{Introduction}

Chronic osteomyelitis is caused by pathogenic microorganisms, which can adhere to - and even invade - mammalian cells. The most common causative organism in osteomyelitis is Staphylococcus aureus. ${ }^{1,2}$ Drug-resistant pathogens such as methicillinresistant $S$. aureus have developed the ability to persist in intracellular locations following drug treatment. ${ }^{3}$ Vancomycin (VCM) is a glycopeptide antibiotic primarily active as an inhibitor of cell wall synthesis in susceptible organisms. ${ }^{4,5}$

At present, the predominant therapies for chronic osteomyelitis are surgery and the local placement of VCM-loaded calcium sulfate beads. ${ }^{6,7}$ Nevertheless, our study on patient samples showed that the local VCM concentration reached a peak at the fourth day postimplantation and then decreased rapidly, indicating unsatisfactory control over the sustained release of drug. ${ }^{8,9}$ Therefore, a new delivery system with controlled and sustained release is crucial. Nanocarriers ${ }^{10,11}$ have attracted prominent interest in the past few decades. Chitosan (CS), a natural and plentiful biopolymer, possesses various biological characteristics; ${ }^{12}$ however, its application has been hampered
Correspondence: Dan Shou Department of Medicine, Zhejiang Medicine, No 132, Tianmushan Road, Hangzhou, Zhejiang 310007, People's Republic of China Email shoudnok@163.com

Ying $\mathrm{Hu}$

Zhejiang Pharmaceutical College, No 888, East Section, Yinxian Main Road, Ningbo, Zhejiang 315100, People's Republic of China Email pharmhawk@I26.com (c)
hereby accept the Terms. Non-commercial uses of the work are permitted without any further permission from Dove Medical Press Limited, provided the work is properly attributed. For permission hereby accept the Terms. Non-commercial uses of the work are permitted without any further permission from Dove Mediect
for commercial use of this work, please see paragraphs 4.2 and 5 of our Terms (https://www.dovepress.com/terms.php). 
largely by its solubility only in acidic environments. ${ }^{13}$ As a result, CS derivatives were synthesized to overcome this defect. Carboxymethyl chitosan (CMC) is a biocompatible material and it is soluble in water without acid. ${ }^{14}$ There were negative charges on the surface of antibiotic-loaded CMC NPs, and burst release of the drug in the initial stage was observed. ${ }^{15}$

In our study, CS was derivatized by N-methylation to $\mathrm{N}, \mathrm{N}, N$-trimethyl chitosan (TMC), a water-soluble compound, to form nanocomplexes with anionic compounds, such as drugs, proteins and DNA, through ionotropic gelation as opposed to a pH-dependent charge. ${ }^{16}$ VCM-loaded TMC NPs (VCM/TMC NPs), prepared in our previous study, ${ }^{17}$ proved to be an effective intracellular mode of drug administration. Additionally, the VCM/TMC NPs had an excellent probability of VCM release in the chronic osteomyelitis rabbit model. However, a burst release phenomenon of VCM was observed. Poly(trimethylene carbonate) (PTMC), ${ }^{18,19}$ a surface-eroding biodegradable material that is fully biocompatible, ${ }^{20}$ exhibits an ideal sustained, zero-order release profile and promotes bone regeneration ${ }^{21}$ due to its unique degradation behavior. ${ }^{22}$

In this study, with the aim to increase the sustained effectiveness of intracellular antibiotics and the probability of biocompatibility, VCM/TMC NP-PTMC was prepared using a composite of VCM/TMC NPs and PTMC. Scanning electron microscopy (SEM) was performed to characterize the surface of the beads before and after erosion. Dialysis was performed to evaluate the drug release, which was measured by reverse-phase high-performance liquid chromatography (HPLC). In vitro cytology examination and the effect on antibacterial activity were analyzed for the NPs. Additionally, the VCM/TMC NP-PTMC was implanted into a chronic osteomyelitis rabbit model to evaluate its antibiotic activity, biocompatibility and bone regeneration ability.

\section{Materials and methods Materials}

PTMC was purchased from Shanghai Leon Chemical Co., Ltd (Shanghai, China). VCM-HCl was supplied by Dalian Meilun Biology Technology Co., Ltd (Dalian, China). Lipase solution from Thermomyces lanuginosus $(100,000 \mathrm{U} / \mathrm{g})$ was obtained from Sigma-Aldrich (St Louis, MO, USA) and used as received. Tetrahydrofuran (THF) and anhydrous hexane were purchased from Tianjin Shield Specialty Chemical Co., Ltd (Zhejiang, China). Beyotime Biotechnology Ltd Co (Shanghai, China) supplied the Cytotoxicity Assay Kit and all cell culture reagents. Methanol (HPLC grade) was purchased from Fisher Chemical (Suzhou, China). Other reagents and chemicals were of analytical reagent grade, unless indicated otherwise.

\section{VCM analysis}

The antibiotic concentration of VCM was determined using our established method ${ }^{23}$ in order for calculating the antibiotic efficiency of VCM/TMC NP-PTMC.

\section{Preparation and characterization of VCM/ TMC NP-PTMC}

VCM/TMC NPs were prepared as described previously. ${ }^{17}$ VCM/TMC NP-PTMC was prepared according to the reported literature with a slight improvement. ${ }^{22}$ In short, PTMC solution was prepared in a glass dish sealed by a cover by dissolving approximately $0.67 \mathrm{~g}$ of polymer in $25 \mathrm{~mL}$ THF while stirring at room temperature until complete dissolution. Then, under continuous stirring, $128 \mathrm{mg}$ of lyophilized VCM/TMC NPs was slowly added and aged for $24 \mathrm{~h}$ to obtain a relatively uniform paste. The obtained suspension was precipitated with an excess of anhydrous hexane and then immediately dried in vacuum overnight. For preparing the test sample of the drug delivery system, the collected dried material was further cut into cuboids - with a square shape of $10 \mathrm{~mm}$ length, $10 \mathrm{~mm}$ breadth and a height of $2 \mathrm{~mm}$ - and stored in a desiccator. VCM-PTMC was prepared in the same way as the experimental control group, and the drug loading was calculated.

The surface topography and morphology of VCM/ TMC NP-PTMC and VCM-PTMC (used as a control) were examined by SEM (S-3400N; Hitachi, Tokyo, Japan). The resulting samples were cut into smaller pieces, placed on the copper cylinders and coated with gold by an ion sputterer. The presence of VCM or VCM/TMC NPs in the PTMC was confirmed through X-ray diffraction (XRD) (X'Pert PRO $\mathrm{X}$-ray diffractometer; PANalytical, Mainz, Germany) and Fourier transform infrared (FTIR) spectroscopy analysis.

\section{In vitro biodegradation study}

The biodegradation behavior of VCM/TMC NP-PTMC and VCM-PTMC (used as a control) was examined in an incubator shaker under sink conditions at $37^{\circ} \mathrm{C}$. In brief, the prepared specimens were suspended in a vial of $5 \mathrm{~mL}$ phosphate-buffered saline (PBS) pH 7.4 ( $\mathrm{NaCl}: 8 \mathrm{~g} / \mathrm{L}, \mathrm{KCl}$ : $0.4 \mathrm{~g} / \mathrm{L}, \mathrm{KH}_{2} \mathrm{PO}_{4}: 0.4 \mathrm{~g} / \mathrm{L}$ and $\mathrm{Na}_{2} \mathrm{HPO}_{4}: 2.86 \mathrm{~g} / \mathrm{L}$ ) or lipase aqueous solution (containing $2 \%$ enzyme concentrate, $0.5 \%$ $\mathrm{CaCl}_{2}$ and $25 \%$ propylene glycol, w/w), closed tightly and rotated at $100 \mathrm{rpm}$. At predetermined time points, square 
specimens were collected and vacuum-dried for $0.5 \mathrm{~h}$. Afterward, the masses were measured in triplicate, and the changes to the surface morphology during the degradation process were examined by SEM. The relative mass represents the degradation ratio and was calculated as Relative mass = $\left(W_{0}-W_{\mathrm{i}}\right) / W_{0}$, where $W_{\mathrm{i}}$ and $W_{0}$ represent the weight of dried specimen before and after incubation in medium.

\section{In vitro release profile}

A dialysis technique with two kinds of release media was used to evaluate the in vitro release profile of VCM from VCM/TMC NP-PTMC. One randomly chosen prepared specimen $(8.39 \pm 1.15 \mathrm{mg})$ was placed in $1 \mathrm{~mL}$ of release medium and then transferred into a dialysis bag (molecular weight cutoff [MWCO]: $2 \mathrm{kDa}$ ), which was dialyzed against $4 \mathrm{~mL}$ of the corresponding medium (PBS 7.4 or lipase aqueous) at $100 \mathrm{rpm}$ in a $37^{\circ} \mathrm{C}$ water bath. On days $2,5,7,12,18$, 27,32 and $36,400 \mu \mathrm{L}$ of the outside solution was harvested, and an equal amount of PBS 7.4 or lipase aqueous solution was supplemented to maintain a constant volume. The amount of released drug was then determined and calculated as the cumulative percentage release.

\section{Antibacterial activity assay}

\section{Bacterial culture}

Gram-positive S. aureus (American Type Culture Collection [ATCC] 6538; Shanghai BioRc Co., Ltd, Shanghai, China) was purchased and cultured in Luria-Bertani (LB) broth at $37^{\circ} \mathrm{C}$ overnight until the optical density of the culture medium reached 0.5 at $600 \mathrm{~nm}$, which was when the content of bacteria reached approximately $10^{8}$ colony-forming units $(\mathrm{CFU}) / \mathrm{mL}$.

\section{Diameter of inhibition zone (DIZ) test}

To ensure the antibacterial effects of VCM released from VCM/ TMC NP-PTMC against $S$. aureus, zones of growth inhibition of the test bacteria were measured by an agar disk diffusion assay. In short, an additional $25 \mathrm{~g}$ of agar per liter was added to $\mathrm{LB}$ liquid medium $(10 \mathrm{~mL})$ with $10 \mu \mathrm{L}$ of stored bacterial suspension, and $100 \mu \mathrm{g} / \mathrm{mL}$ of VCM solution served as the control. Furthermore, sterile filter disks (6 $\mathrm{mm}$ in diameter) were impregnated with $100 \mu \mathrm{L}$ of PBS or lipase release aliquots or control solutions for approximately $30 \mathrm{~min}$ plated on the surface of LB agar plates (each plate contained four paper disks), and these were subsequently incubated in a biochemical incubator (SHP-250; Shanghai Senxin Test Instrument Co., Ltd, Shanghai, China) with an atmosphere of $5 \% \mathrm{CO}_{2}$ at $37^{\circ} \mathrm{C}$ for $24 \mathrm{~h}$. The antimicrobial activity was evaluated by measuring the DIZ around the disk. The DIZ values were taken in four perpendicular directions and measured by a Vernier caliper. All tests were performed in triplicate.

\section{Antibacterial activity on the infected rat calvarial osteoblasts (ROBs)}

ROBs were isolated from neonatal (2-day-old) Sprague Dawley rats: $;{ }^{24}$ cells at passage four were used. To identify the infected ROBs, S. aureus was labeled with fluorescein isothiocyanate (FITC; $100 \mu \mathrm{g} / \mathrm{mL}$ ) for $4 \mathrm{~h}$. The nuclei of the ROBs were labeled with 4',6-diamidino-2-phenylindole, and the cytoskeleton was labeled with rhodamine phalloidin, following a modified previous protocol. ${ }^{25} \mathrm{An} S$. aureus suspension (bacterial cells corresponded to approximately $1 \times 10^{6} \mathrm{CFU} / \mathrm{mL}$ ) was added to induce intracellular infection. The cells were incubated for $2 \mathrm{~h}$ in a $\mathrm{CO}_{2}$-free incubator, and then $\mathrm{VCM} /$ TMC NP-PTMC or VCM-PTMC (the VCM concentration was $50 \mu \mathrm{g} / \mathrm{mL}$ ) was added. Untreated ROBs were the control group. The number of bacterial colonies was quantified using a medical image management system (Image-Pro Plus, IPP6.0; Media Cybernetics, Inc., Bethesda, MD, USA).

\section{Protein adsorption}

Bicinchoninic acid (BCA) assay was performed to quantify the total protein adsorption onto the VCM/TMC NP-PTMC $(5 \times 5 \times 2 \mathrm{~mm}$ with similar weight), as reported by Shalumon et al, ${ }^{26}$ with small change. Briefly, VCM/TMC NP-PTMC and VCM-PTMC (used as a control) were placed in a 24-well plate and incubated at $37^{\circ} \mathrm{C}$ with Dulbecco's Modified Eagle's Medium (DMEM, pH 7.4) supplemented with $10 \%$ $(\mathrm{v} / \mathrm{v})$ heat-inactivated fetal bovine serum (FBS) for $24 \mathrm{~h}$ and $48 \mathrm{~h}$. The total amount of protein in the medium was determined prior to adding the materials. At the preestablished time, according to the manufacturer's guidelines (Beyotime Biotechnology Ltd Co.,), the supernatants were collected to determine the remains in the medium using the following equation. Protein adsorption was expressed as a percentage content of the control cells.

$$
\mathrm{R}(\%, \mathrm{w} / \mathrm{w})=(\mathrm{Mp}-\mathrm{Sp}) / \mathrm{Mp} \times 100
$$

where $\mathrm{R}, \mathrm{Mp}$ and $\mathrm{Sp}$ represent the protein adsorption ratio, total amount of protein in medium, and amount of protein in supernatants, respectively.

\section{Cell proliferation}

The EtO-sterilized VCM/TMC NP-PTMC and VCM-PTMC (in triplicate) were tested for osteoblast proliferation by 
MTT (3-(4,5-dimethylthiazol-2-yl)-2,5-diphenyltetrazolium bromide) assay, as reported earlier. ${ }^{27}$ The osteoblasts were seeded on the sterile VCM/TMC NP-PTMC and VCMPTMC at a density of $5 \times 10^{3}$ cells per well. DMEM was added into each well, and the plates were incubated in $\mathrm{C}_{2}$-free incubator. After 4 days and 7 days of incubation, the beads were taken out and put into a new plate, and $10 \mu \mathrm{L}$ MTT ( $5 \mathrm{mg} / \mathrm{mL}$, dissolved in MTT assay solvent) was added to each well and the cells were further incubated for $4 \mathrm{~h}$. After incubation, $100 \mu \mathrm{L}$ of formazan dissolution buffer was added to each well to dissolve the MTT formazan crystals. The plates were shaken for $5 \mathrm{~min}$ and the absorbance was measured at $490 \mathrm{~nm}$ using a microplate reader. The results of five repeated experiments were expressed as a percentage viability of the control cells.

\section{In vivo treatment in a chronic osteomyelitis rabbit model}

Animal groups and treatment

All the animal experiments were performed in accordance with the Guide for the Care and Use of Laboratory Animals and were approved by the Bioethics Committee of Zhejiang Academy of Traditional Chinese Medicine. Male New Zealand White rabbits (SCXK 2014-0001), aged 3 months and weighing $3.0 \pm 0.2 \mathrm{~kg}$, were housed in individual cages under air-controlled conditions $\left(20^{\circ} \mathrm{C} \pm 1^{\circ} \mathrm{C}\right.$ and $12 \mathrm{~h} / 12 \mathrm{~h}$ light-dark illumination cycles). The model was prepared according to a previous report, ${ }^{28}$ and five rabbits were verified by microbiological and histological examination at the fourth week after infection. The model rabbits were divided into the model, VCM/TMC NP-PTMC and VCM-PTMC groups. The two test groups were implanted with VCM/TMC NP-PTMC or VCM-PTMC beads (10 mg VCM per rabbit) after thoroughly cleaning the infected tissue. The rabbits in the model group were housed under the same conditions but not treated.

\section{Antibacterial activity evaluation}

At the end of the fourth week after infection and at the second, fourth and sixth weeks after treatment, inflammatory indexes in serum, such as highly sensitive C-reactive protein (CRP) and white blood cell count (WBC), were determined. At the end of the sixth week after treatment, the rabbits were euthanized with overdosages of pentobarbital sodium. The marrow in the tibia was spread on top of sheep blood agar plates, incubated overnight at $37^{\circ} \mathrm{C}$ and the number of colonies counted.

\section{Bone repair capability evaluation}

At the end of the fourth and eighth weeks after treatment, rabbits were euthanized with overdosages of pentobarbital sodium, and the tibia specimens were examined by microcomputed tomography (micro-CT) (SkyScan 1172 micro-CT scanner; Bruker, Zurich, Switzerland). Scores for the ratio of bone volume/tissue volume (BV/TV), as well as the bone mineral density (BMD), were obtained directly from the 3D model. The tibia specimens were subjected to hematoxylinand-eosin staining and immunohistochemical analysis at the end of the fourth and eighth weeks. Alizarin red $(30 \mathrm{mg} / \mathrm{kg})$ and calcein $(20 \mathrm{mg} / \mathrm{kg})$ were intraperitoneally injected into the rabbits at 7 days and 14 days before euthanization to measure the dynamic parameters of bone such as the mineral appositional rate (MAR) and bone formation rate (BFR). ${ }^{29}$

\section{Statistical analysis}

The software program GraphPad Prism 5 (GraphPad Software Inc, La Jolla, CA, USA) was used for statistical analysis. The data were presented as the mean \pm SD for all results. The statistical significance was determined either by a paired $t$-test or a one-way analysis of variance (ANOVA) with the Bonferroni or Dunnett's post hoc tests, depending on the experimental setup. A value of $P<0.05$ (two-tailed) was considered to be statistically significant.

\section{Results}

\section{Characterization of VCM/TMC NP-PTMC}

The SEM images (Figure 1A) of VCM-PTMC and VCM/ TMC NP-PTMC showed that the NPs were spherical in shape with no obvious particle aggregation in the VCM/TMC NP-PTMC sample. The surface of the spherical VCM/TMC was altered by the presence of VCM/TMC NPs. The surface erosion of these two drug delivery systems showed that in PBS at $\mathrm{pH} 7.4$, the matrix degradation phenomenon was more obvious in VCM-PTMC than in VCM/TMC NP-PTMC after the 5-day incubation. During the enzymatic degradation process, an obvious degradation of the PTMC structure was seen after 3 days of incubation, while the matrix degradation of VCM/TMC NP-PTMC was not obvious.

The XRD spectrum (Figure 1B) showed that VCM/TMC NPs were amorphous in nature. In the XRD patterns of VCM/ TMC NP-PTMC and PTMC, the peaks at $2 \theta=21.0^{\circ}$ are the characteristic peaks of PTMC, corresponding to a layered structure with a basal spacing of $0.42 \mathrm{~nm}$ (calculated by Bragg's diffraction formula). ${ }^{30}$ The peaks disappear completely for the VCM/TMC NPs.

The infrared (IR) spectrum of VCM/TMC NP-PTMC microspheres (Figure 1C) showed that at 3300-3450 $\mathrm{cm}^{-1}$, the stretch vibration absorption spectra of $-\mathrm{OH}$, and 2900-2800 $\mathrm{cm}^{-1}$, the asymmetric and symmetric stretch vibration absorption spectra of $-\mathrm{CH}_{2}-$ in $\mathrm{TMC}$, both 

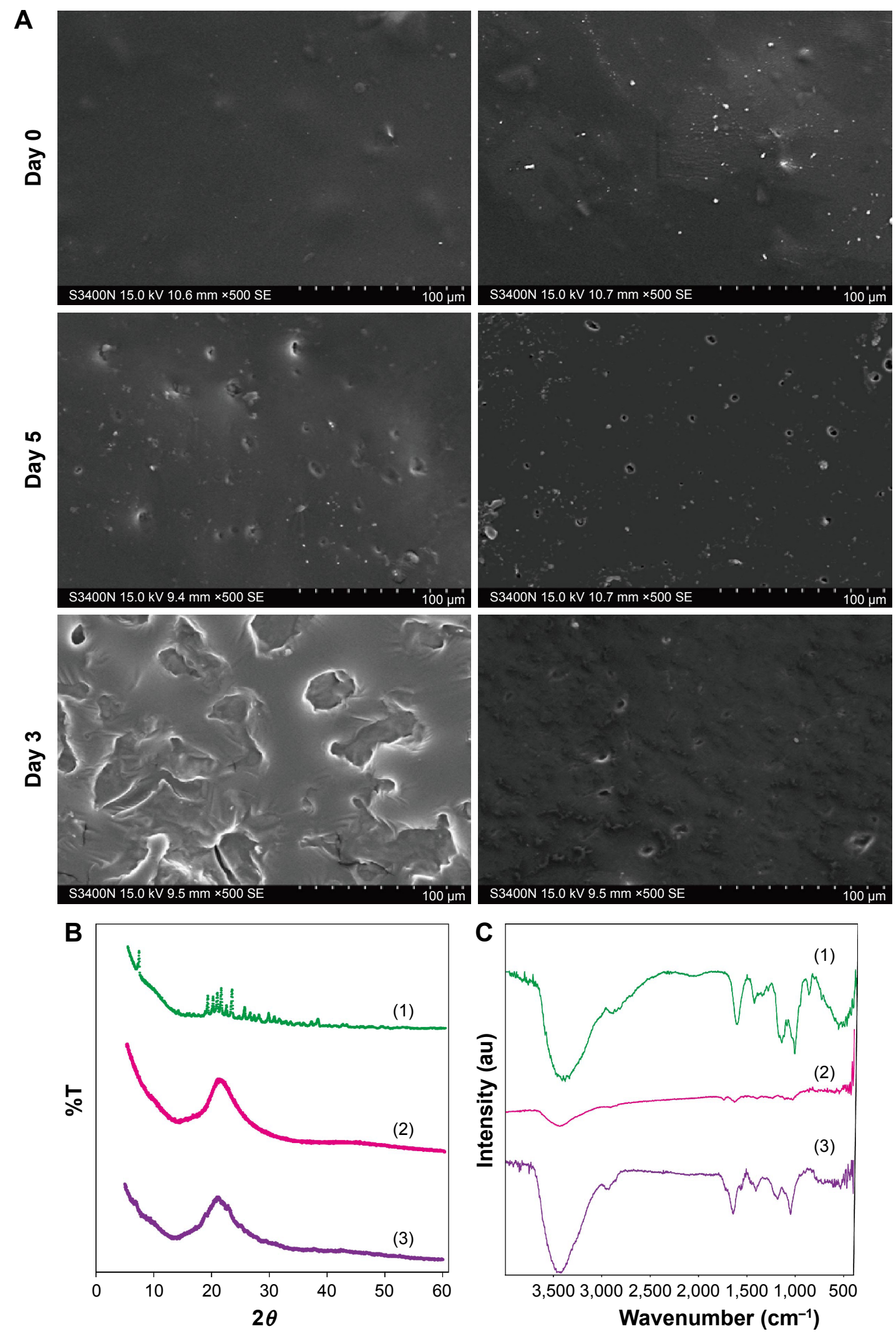

Figure I The characterization of VCM-PTMC and VCM/TMC NP-PTMC.

Notes: (A) SEM images of the surfaces of VCM-PTMC (left) and VCM/TMC NP-PTMC (right) before and after incubation, as seen after incubation in PBS 7.4 and lipase aqueous medium at $37^{\circ} \mathrm{C}$. The top images show the surfaces of the beads before incubation, the middle images show the surfaces of the beads after 5 days of incubation in PBS 7.4, and the bottom images show the exposure to the surfaces of the beads after 3 days of incubation in lipase aqueous medium. (B) XRD spectra of VCM/PTMC and VCM/TMC NP-PTMC. (C) FTIR spectra of VCM-PTMC and VCM/TMC NP-PTMC. (I) VCM-TMC NPs; (2) PTMC; and (3) VCM/TMC NP-PTMC.

Abbreviations: FTIR, Fourier transform infrared spectroscopy; NP, nanoparticle; PBS, phosphate-buffered saline; PTMC, poly(trimethylene carbonate); SEM, scanning electron microscopy; TMC, N-trimethyl chitosan; VCM, vancomycin; XRD, X-ray diffraction. 
appeared. Compared to the IR spectrum of VCM-TMC NPs, a new band at $1747 \mathrm{~cm}^{-1}$, attributed to the $\mathrm{C}-\mathrm{O}$ stretching region, appeared in the spectrum of the PTMC. The region between $1500 \mathrm{~cm}^{-1}$ and $1360 \mathrm{~cm}^{-1}$ is characterized by the $1475 \mathrm{~cm}^{-1} \mathrm{CH}_{3}$ band, which is attributed to the asymmetrical stretching of $\mathrm{C}-\mathrm{H}$ in the methyl groups on the quaternary ammonium, and this appeared in the spectrum of TMC. The $1380 \mathrm{~cm}^{-1}$ and $1360 \mathrm{~cm}^{-1}$ peaks could be ascribed to the $\mathrm{C}-\mathrm{H}$ deformation and asymmetric bands (Figure 1C).

\section{In vitro biodegradation study}

The loading efficiency (LE) of VCM/TMC NP-PTMC was calculated according to the following equation:

$$
\begin{aligned}
\mathrm{LE}(\%, \mathrm{w} / \mathrm{w}) & =\mathrm{Aa} /(\mathrm{Aa}+\mathrm{Ap}) \times 100 \\
& =0.01 /(0.01+0.67) \\
& =1.47
\end{aligned}
$$

where $\mathrm{Aa}$ and Ap represent the total amount of antibiotic and total amount of polymer material, respectively.

PBS at pH 7.4 and lipase aqueous media were applied to determine the in vitro biodegradation. Figure $2 \mathrm{~A}$ depicts that on days 2-6, the VCM-PTMC in the lipase aqueous medium showed a slightly higher relative degradation mass in comparison to the VCM/TMC NP-PTMC, and on the sixth day, $0.19 \pm 0.01$ relative degradation mass was observed for the VCM/TMC NP-PTMC. However, the VCM-PTMC showed $0.35 \pm 0.03$ relative degradation mass in the lipase aqueous medium. In addition, in PBS 7.4, the VCM-PTMC showed higher relative degradation mass compared to the VCM/TMC NP-PTMC, and on the sixth day, $0.59 \pm 0.11$ relative degradation mass was observed for the VCM-PTMC. At the end of the 36th day, the VCM/TMC NP-PTMC showed $0.53 \pm 0.05$ relative degradation mass.

\section{In vitro drug release study}

The cumulative release curves (Figure 2B) showed the release behavior of VCM from VCM-PTMC and VCM/ TMC NP-PTMC over 36 days in PBS 7.4 and lipase aqueous medium. The cumulative release of VCM followed a steady, continued-release pattern, without a burst release phenomenon. The cumulative release rates of VCM from VCMPTMC and VCM/TMC NP-PTMC were $90.79 \% \pm 0.06 \%$ and $83.57 \% \pm 0.02 \%$, respectively, with 36-day incubation in the lipase aqueous medium. The cumulative release rates of VCM from VCM-PTMC and VCM/TMC NP-PTMC were $24.21 \% \pm 0.02 \%$ and $18.71 \% \pm 0.04 \%$ with 36 -day incubation in the PBS 7.4 medium, respectively.

\section{Antibacterial activity}

DIZ results (Figure 2C) showed that the DIZ values of the release solution from VCM-PTMC and VCM/TMCNP-PTMC were $10.84 \pm 0.70 \mathrm{~mm}$ and $11.18 \pm 0.66 \mathrm{~mm}$ on the 18 th day of incubation in lipase aqueous solution. On the 32 nd day, the DIZ value of VCM-PTMC was reduced to $6.23 \pm 0.69 \mathrm{~mm}$ but was $9.93 \pm 0.41 \mathrm{~mm}$ for VCM/TMC NP-PTMC. The DIZ values of release solution from VCM-PTMC and VCM/TMC NP-PTMC incubated in PBS 7.4 were $10.30 \pm 0.36 \mathrm{~mm}$ and $11.06 \pm 0.30 \mathrm{~mm}$ on the 27 th day and $9.45 \pm 0.37 \mathrm{~mm}$ and $9.46 \pm 0.25 \mathrm{~mm}$ on the 36 th day, respectively.

As shown in Figure $2 \mathrm{~F}$ and $\mathrm{G}$, following $2 \mathrm{~h}$ of infection with $S$. aureus, labeled bacteria became visible inside the ROBs, while after $24 \mathrm{~h}$ incubation of infected ROBs with $\mathrm{VCM} / \mathrm{TMC}$ NP-PTMC, the amount of $S$. aureus both inside and outside the cells was drastically reduced. In addition, in infected ROBs incubated with VCM-PTMC, S. aureus visible outside the ROBs was drastically reduced, but $S$. aureus inside the ROBs showed strong density of fluorescence. The results indicated that VCM/TMC NPs inhibited $S$. aureus intracellularly and extracellularly.

\section{Protein adsorption}

The protein adsorption studies were carried out for $24 \mathrm{~h}$ and $48 \mathrm{~h}$, respectively, wherein the VCM/TMC NP-PTMC showed a significantly higher protein adsorption compared to the control beads at both the time periods $(P<0.01)$ (Figure 2D). Additionally, the maximum protein adsorption on the VCM/TMC NP-PTMC was achieved in the initial $48 \mathrm{~h}$ period. The VCM-PTMC showed a significantly higher protein adsorption in the initial $48 \mathrm{~h}$ period than at $24 \mathrm{~h}(P<0.05)$.

\section{Cell proliferation}

Cell proliferation activity was tested by the MTT assay. Figure 2E showed that after 4-day incubation, VCM/TMC NP-PTMC elicited significantly higher cell proliferation activity $(P<0.05)$ compared to VCM-PTMC. Similar results were obtained with significance $(P<0.01)$ after 7 days of incubation.

\section{In vivo antibacterial activity}

Most model rabbits had a large number of infected sinuses, and white and yellow pus overflowed from the wounds (Figure 3A), similar to the representative symptom of chronic osteomyelitis in clinic. Streaking the marrow lysates on agar plates resulted in a concordantly decreased number of colonies for the VCM/TMC NP-PTMC and VCM-PTMC 
groups, which even reduced down to zero for the VCM/ TMC NP-PTMC group after 6-week treatment (Figure 3B). Compared to the control group, the levels of CRP and WBC were higher in the model group. At the end of the fourth week and eighth week after treatment with VCM/TMC NP-PTMC or VCM-PTMC, both antibiotic delivery systems significantly decreased the CRP and WBC (Figure 3D).
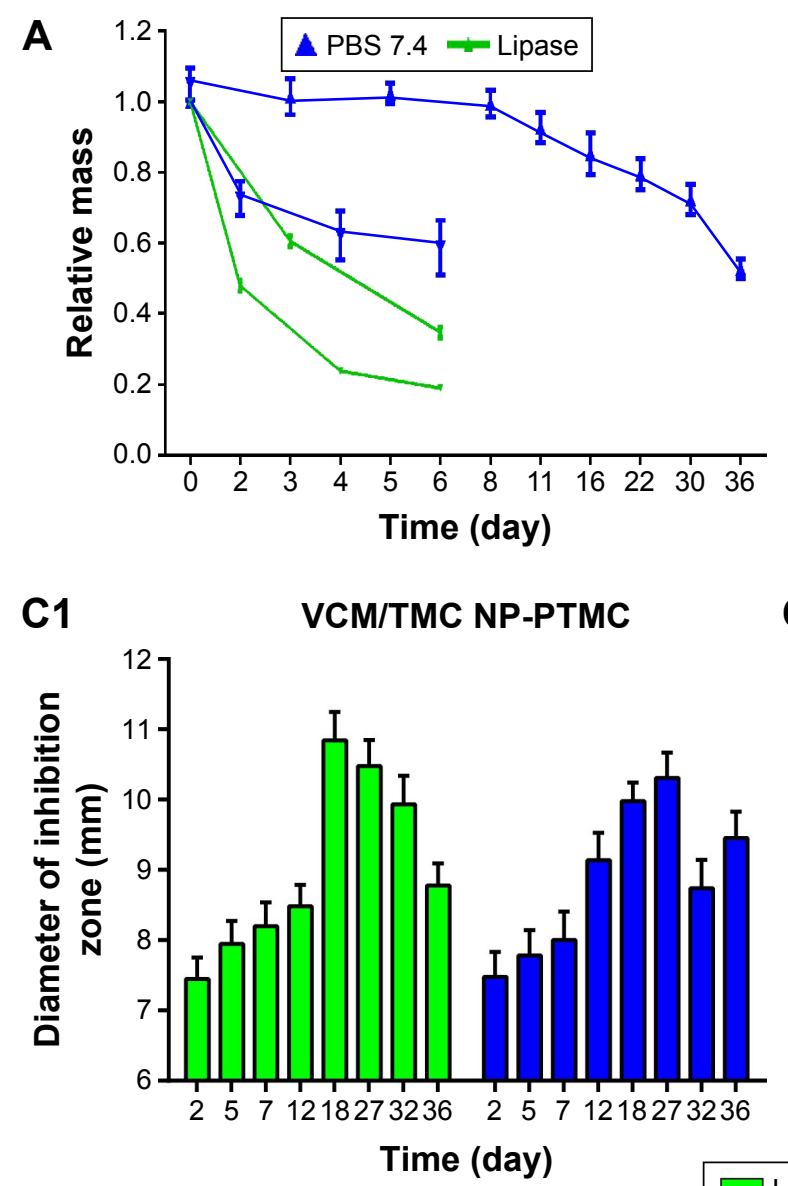

D

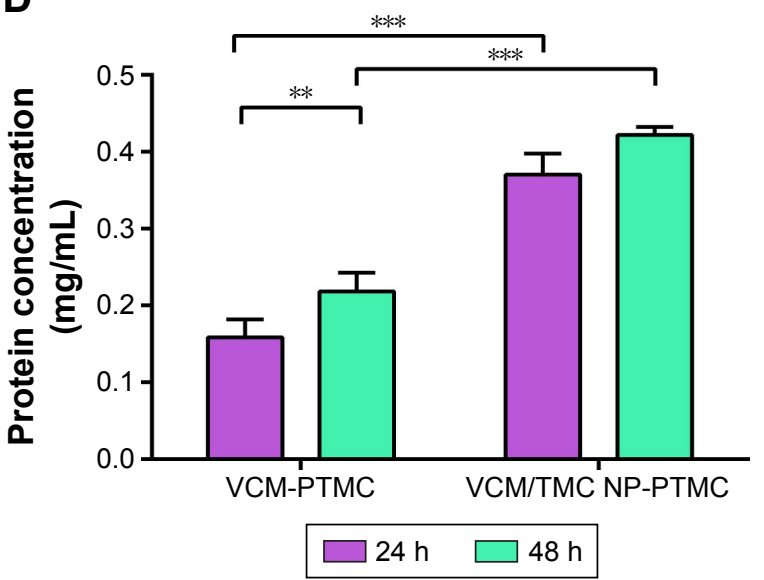

\section{Bone repair capability}

After 8 weeks of treatment, the tibial plateau of the VCM/ TMC NP-PTMC group seemed flatter than that of the VCM-PTMC group (Figure 3C). The images of the 3D reconstruction in Figure 4A indicated a progressive increase in bone volume at the end of eighth week after treatment with VCM/TMC NP-PTMC and VCM-PTMC while it tended

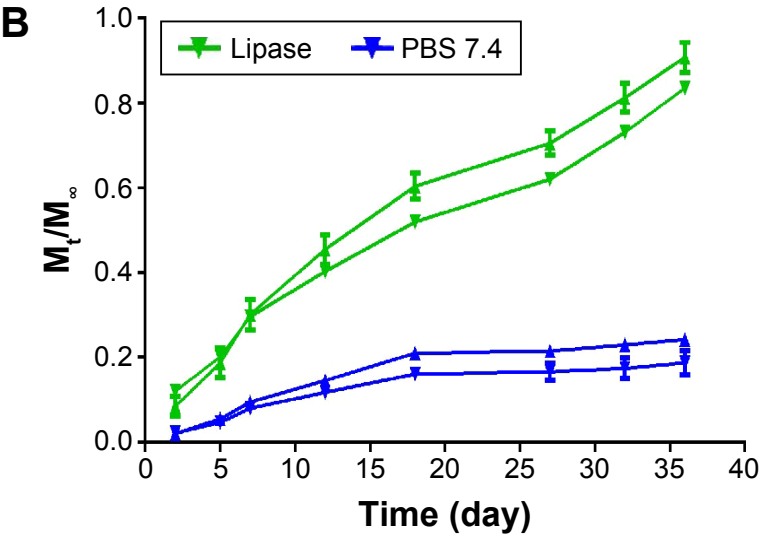

C2

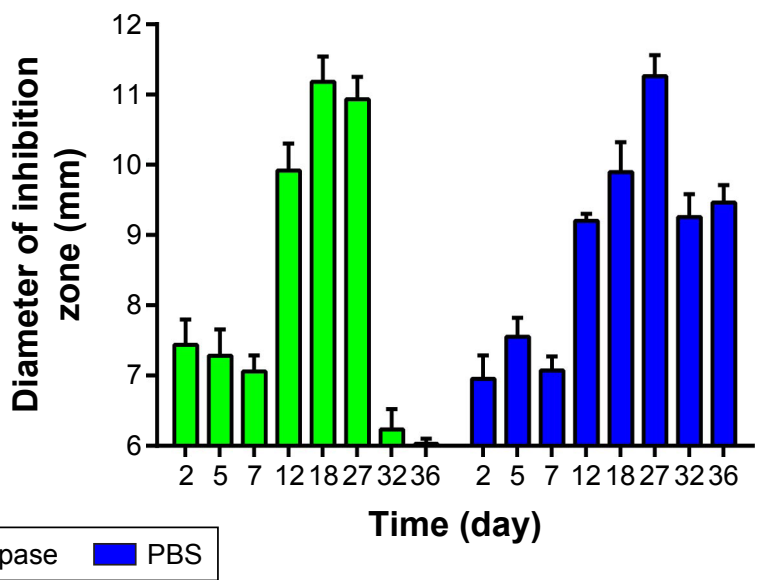

E

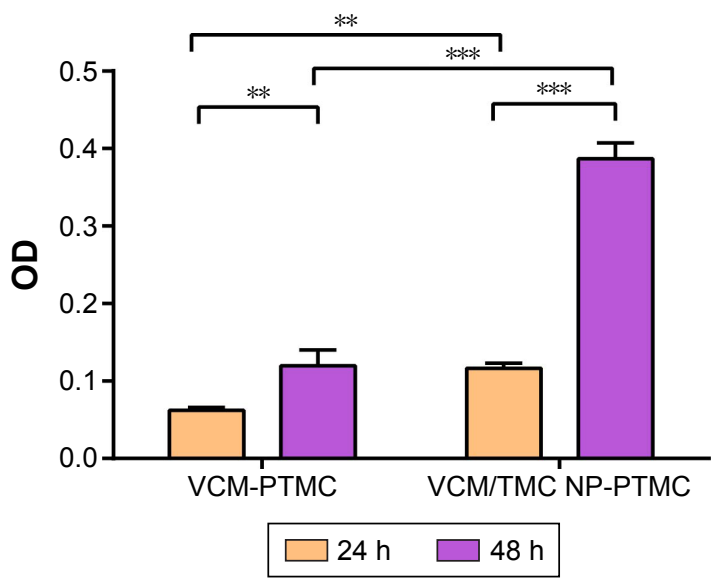

Figure 2 (Continued) 
$\mathbf{F}$

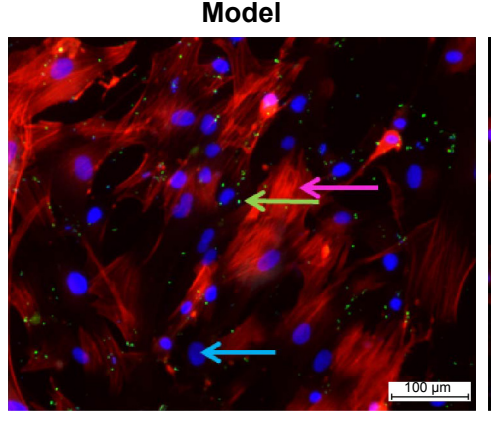

VCM-PTMC

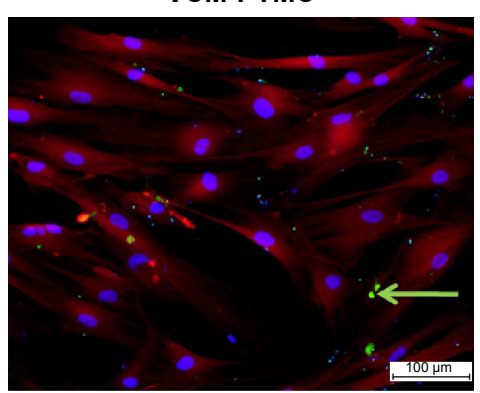

VCM/TMC NP-PTMC

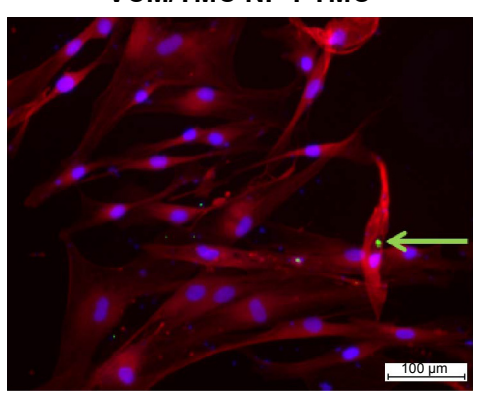

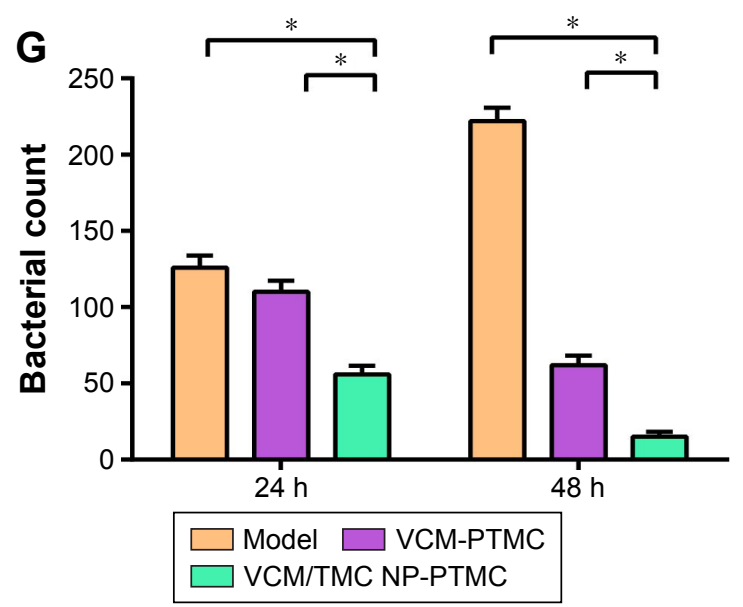

Figure 2 The biodegradation, antibacterial activity, protein adsorption, cell proliferation and drug release characteristics.

Notes: (A) Relative biodegradation mass of VCM-PTMC $(\mathbf{\Delta})$ and VCM/TMC NP-PTMC $(\boldsymbol{\nabla})$ in PBS 7.4 and lipase aqueous media. (B) The curves of cumulative release of VCM from VCM-PTMC $(\mathbf{\Delta})$ and VCM/TMC NP-PTMC $(\boldsymbol{\nabla})$ in the PBS 7.4 and in the lipase aqueous media. (C) The graphical representation of the antibacterial activity of VCM-PTMC and VCM/TMC NP-PTMC solution released from PBS 7.4 and lipase aqueous media. (CI) The antibacterial activity of VCM/TMC NP-PTMC solution released from PBS 7.4 and lipase media; (C2) The antibacterial activity of VCM-PTMC solution released from PBS 7.4 and lipase media. (D) The graphical representation of protein adsorption activity of VCM-PTMC and VCM/TMC NP-PTMC. **P $<0.05$; ***P $<0.01$. (E) The graphical representation of attachment and proliferation ability on the surface of VCM-PTMC and VCM/ TMC NP-PTMC. $* * P<0.05 ; * * * P<0.01$. (F) Fluorescence optical images of fluorescently stained osteoblastic cell nuclei (blue arrow) and cytoskeletal F-actin (pink arrow), invaded by Staphylococcus aureus (green arrow). (G) The bacterial count after $24 \mathrm{~h}$ and $48 \mathrm{~h}$ incubation of infection ROBs with VCM-PTMC and VCM/TMC NP-PTMC. *P $<0.05$.

Abbreviations: NP, nanoparticle; OD, optical density; PBS, phosphate-buffered saline; PTMC, poly(trimethylene carbonate); ROBs, rat calvarial osteoblasts; TMC, N-trimethyl chitosan; VCM, vancomycin.

toward bone loss in the model groups. The micro-CT indexes (Figure 4B) showed that the BV/TV and BMD decreased further and were significantly lower in the model group than in the VCM/TMC NP-PTMC and VCM-PTMC groups. In addition, the BV/TV and BMD were markedly higher in the VCM/TMC NP-PTMC group than in the VCM-PTMC group. A histomorphometric analysis showed a significant difference between the VCM/TMC NP-PTMC and VCM-PTMC groups, demonstrated by increased MAR and BFR (Figure 4C and D). These results suggested the effect of VCM/TMC NP-PTMC on promoting bone healing. Infection caused a decrease in the number of bone trabeculae, but the VCM/TMC NP-PTMC and VCM-PTMC groups showed less pronounced decreases than the model group. A pronounced decrease in number of osteoblasts per trabecular bone area (NOb/TAr) and number of osteoblasts per bone perimeter $(\mathrm{NOb} / \mathrm{Bpm})$ was induced by $S$. aureus, but this decrease was mitigated by VCM/TMC NP-PTMC and VCM-PTMC treatment (Figure 5A and B).
As shown in Figure 5C, significantly decreased rates of integrated optical density/area (IOD/area) for osteocalcin (OC) were observed in the model group than in both VCM/ TMC NP-PTMC and VCM-PTMC groups, which was more pronounced in the VCM/TMC NP-PTMC group.

\section{Discussion}

In our previous study, ${ }^{17}$ the geometric mean diameter of VCM/TMC NPs obtained from atomic force microscopy was 200-325 nm, mean size distribution was 220-230 nm with a fairly monodisperse polydispersity index, which was almost $<0.2$; meanwhile, VCM/TMC NPs carried a positive zeta potential of $14.6 \pm 0.8 \mathrm{mV}$ at physiological $\mathrm{pH}$, suggesting that these NPs are probably ionically stabilized against particle agglomeration due to the positive charges on their surface. In this study, the SEM results illustrated the presence of spherical VCM/TMC NPs with a smooth surface and compact structure. The X-ray and IR spectrum results 
A

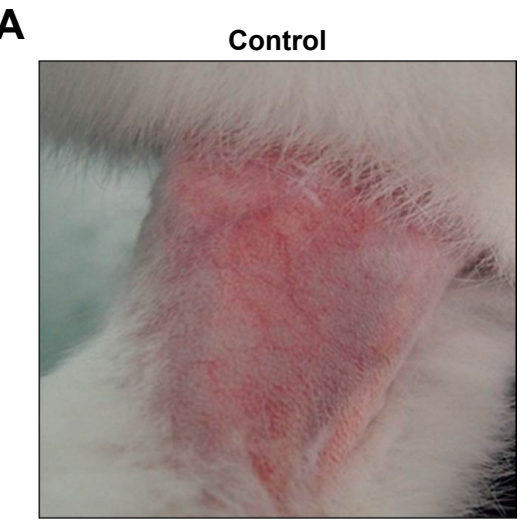

C

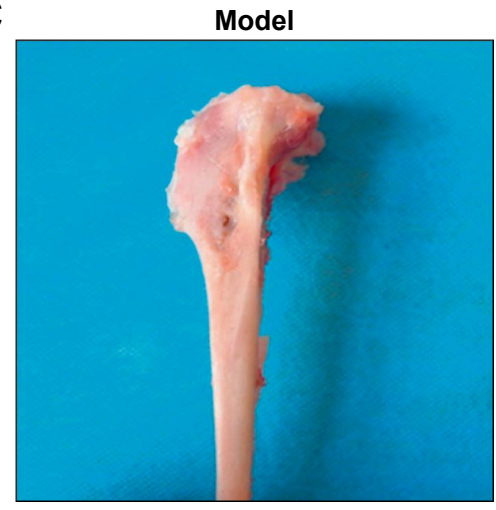

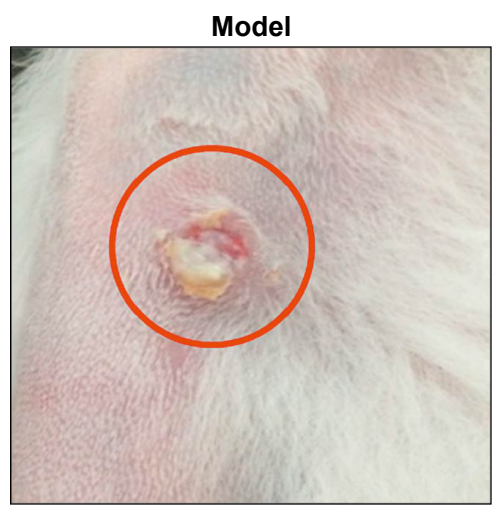

VCM-PTMC

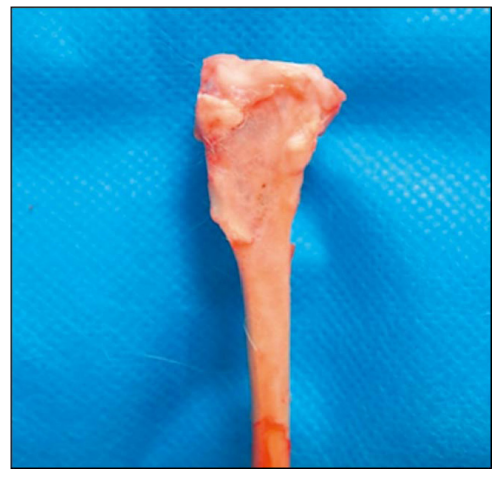

B
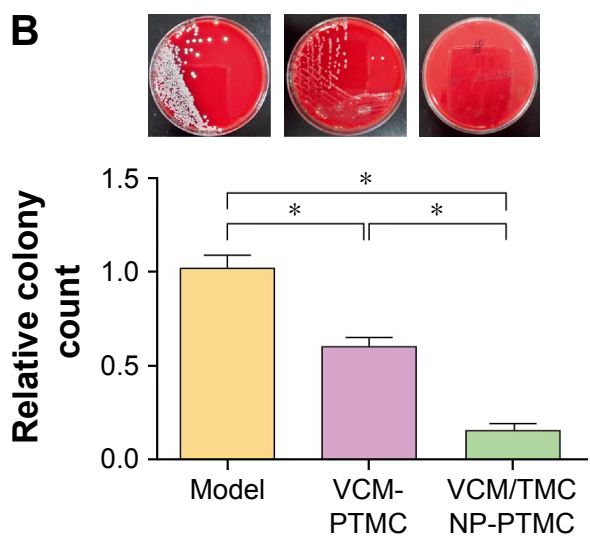

VCM/TMC NP-PTMC

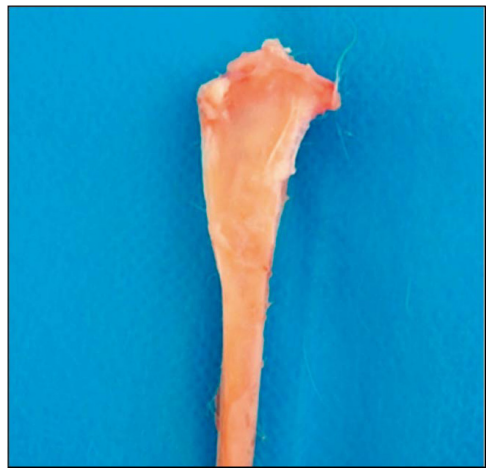

D
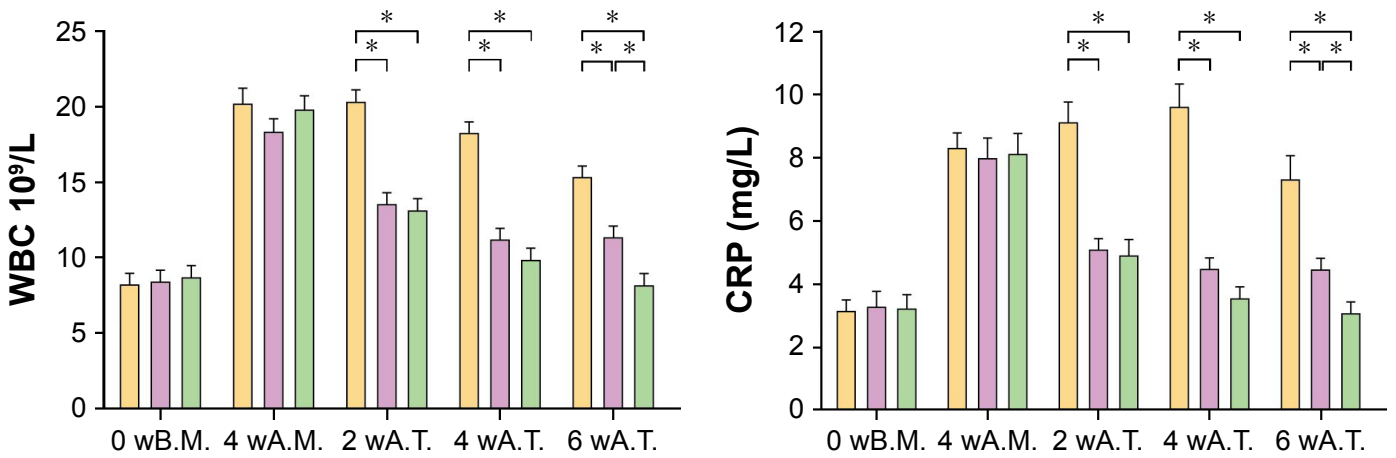

$\square$ Model $\square$ VCM-PTMC $\square$ VCM/TMC NP-PTMC

Figure 3 The antibacterial activity of VCM-PTMC and VCM/TMC NP-PTMC.

Notes: (A) Appearance of the rabbit legs at the fourth week after infection with Staphylococcus aureus. (B) Typical photograph of bacterial colonies forming on sheep blood agar plates, and the number of bacterial colonies in the tibia marrow counted following overnight incubation. (C) Typical photographs of tibia that underwent 8 weeks of treatment with VCM-PTMC and VCM/TMC NP-PTMC. (D) The results of WBC and CRP estimations in the rabbit serum at the time points of before modeling, fourth week after infection, fourth week after treatment and eighth week after treatment. $* P<0.05$.

Abbreviations: 0 wB.M., 0 weeks before modeling; 4 wA.M., 4 weeks after modeling; 2 wA.T., 2 weeks after treatment; 4 wA.T., 4 weeks after treatment; 6 wA.T., 6 weeks after treatment; CRP, C-reactive protein; NP, nanoparticle; PTMC, poly(trimethylene carbonate); TMC, N-trimethyl chitosan; VCM, vancomycin; WBC, white blood cell.

showed that VCM/TMC NPs are successfully introduced to the PTMC.

VCM-PTMC had more rapid biodegradation than VCM/TMC NP-PTMC, which is due to the slow degradation of VCM/TMC NPs in the VCM/TMC NP-PTMC. Biodegradable polymers can be arbitrarily classified into two groups: bulk eroding (homogeneous) and surface eroding (heterogeneous) polymers. ${ }^{22}$ PTMC biodegradation only occurs on the surface; therefore, its degradation process is slower than materials degraded through bulk erosion, which prevents the bulk erosion of the VCM/TMC NPs due to immediate contact with the solvent and premature release. ${ }^{31}$ In addition, compared to the PBS pH 7.4 medium, both delivery systems had obvious swelling phenomenon in the lipase aqueous medium, which is associated with the conformation of the adsorbed enzymes on the surface. As the PTMC 
A
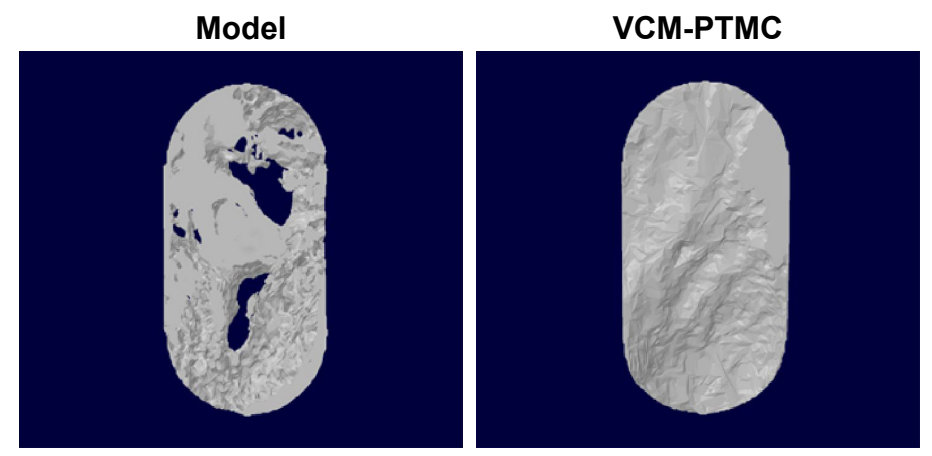

B
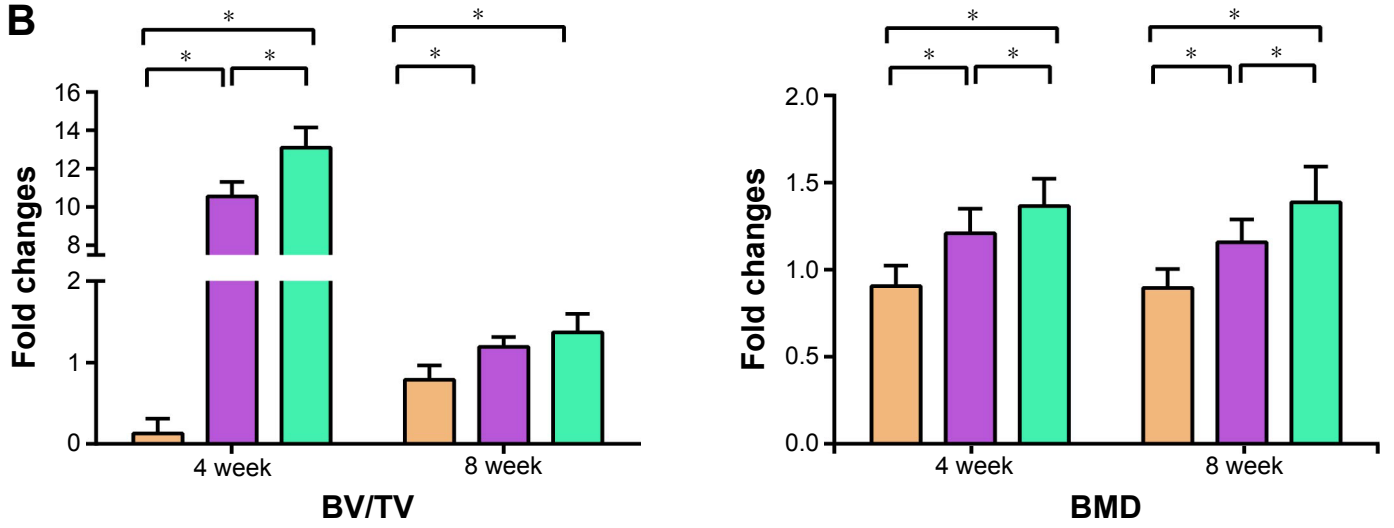

$\square$ Model $\square$ VCM-PTMC $\square$ VCM/TMC NP-PTMC

C
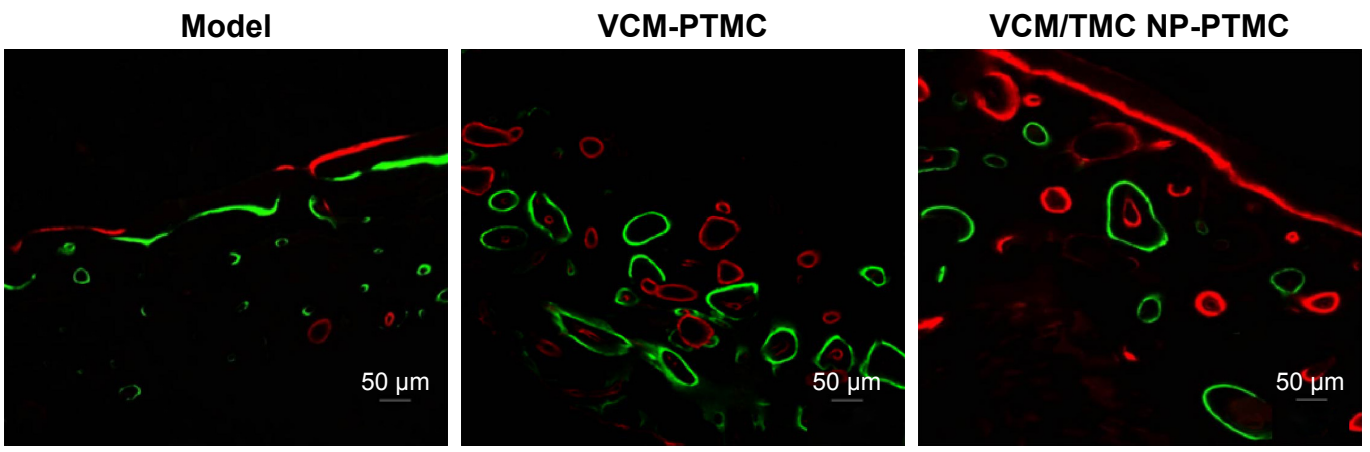

D
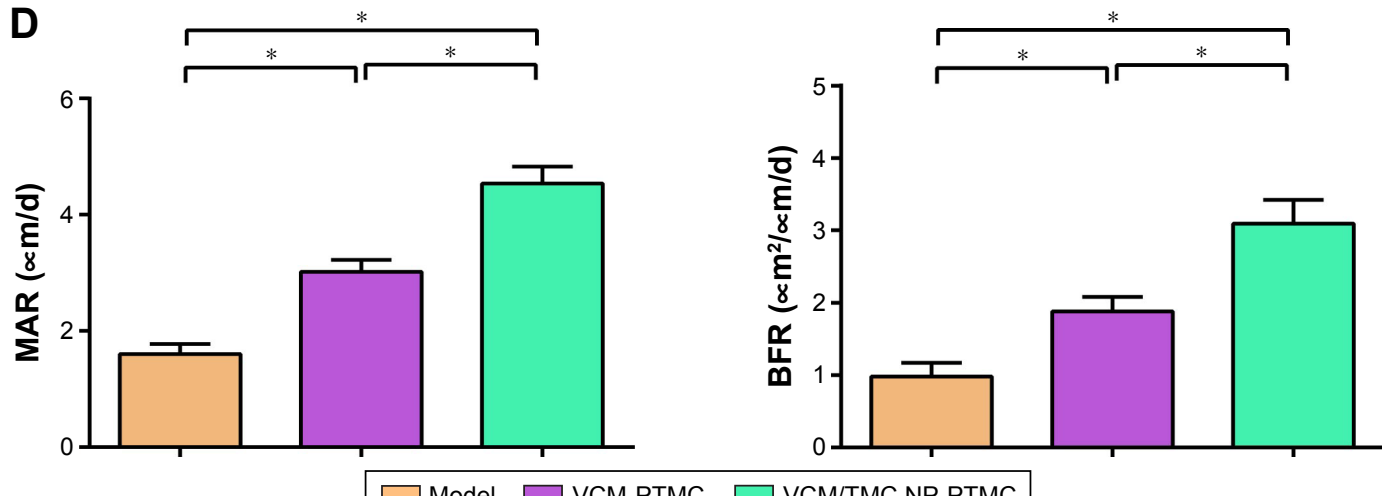

Figure 4 The bone repair capability of VCM-PTMC and VCM/TMC NP-PTMC.

Notes: (A) Micro-CT morphometry of bone defect of the tibia in chronic osteomyelitis model rabbits that underwent 8 weeks of treatment with VCM-PTMC and VCM/ TMC NP-PTMC. (B) The columns represent the scores of the BV/TV and BMD from five rabbits per group. (C) Typical photographs of fluoroscope observation on tibia with double-fluorescent labeling. (D) The columns represent the scores of MAR and BFR. $* P<0.05$.

Abbreviations: BFR, bone formation rate; BMD, bone mineral density; BV/TV, bone volume/tissue volume; CT, computed tomography; MAR, mineral appositional rate; NP, nanoparticle; PTMC, poly(trimethylene carbonate); TMC, N-trimethyl chitosan; VCM, vancomycin. 


\section{A}
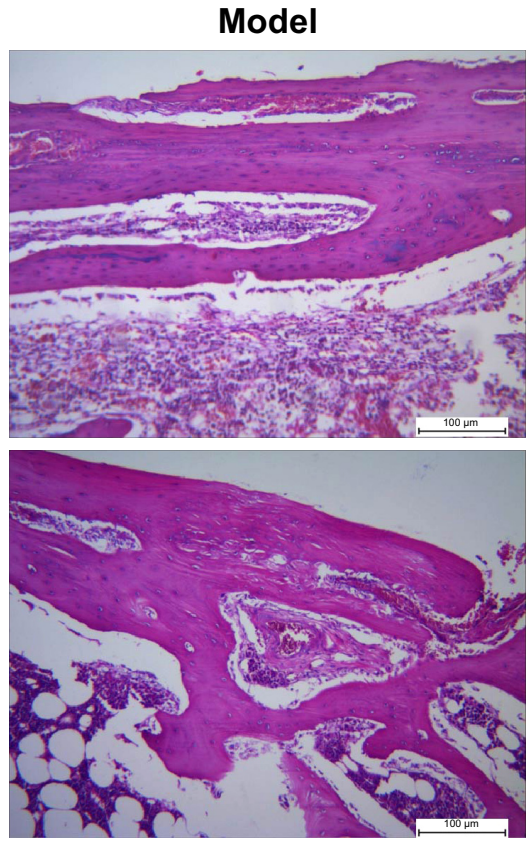

VCM-PTMC
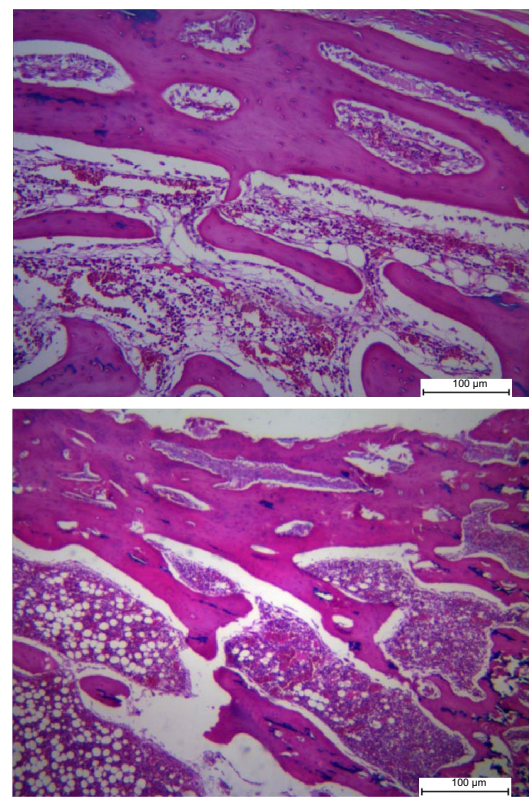

VCM/TMC NP-PTMC
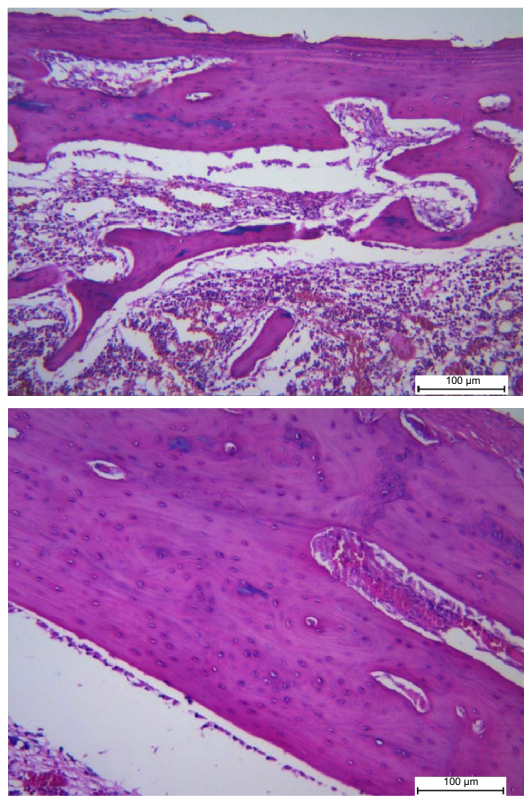

B
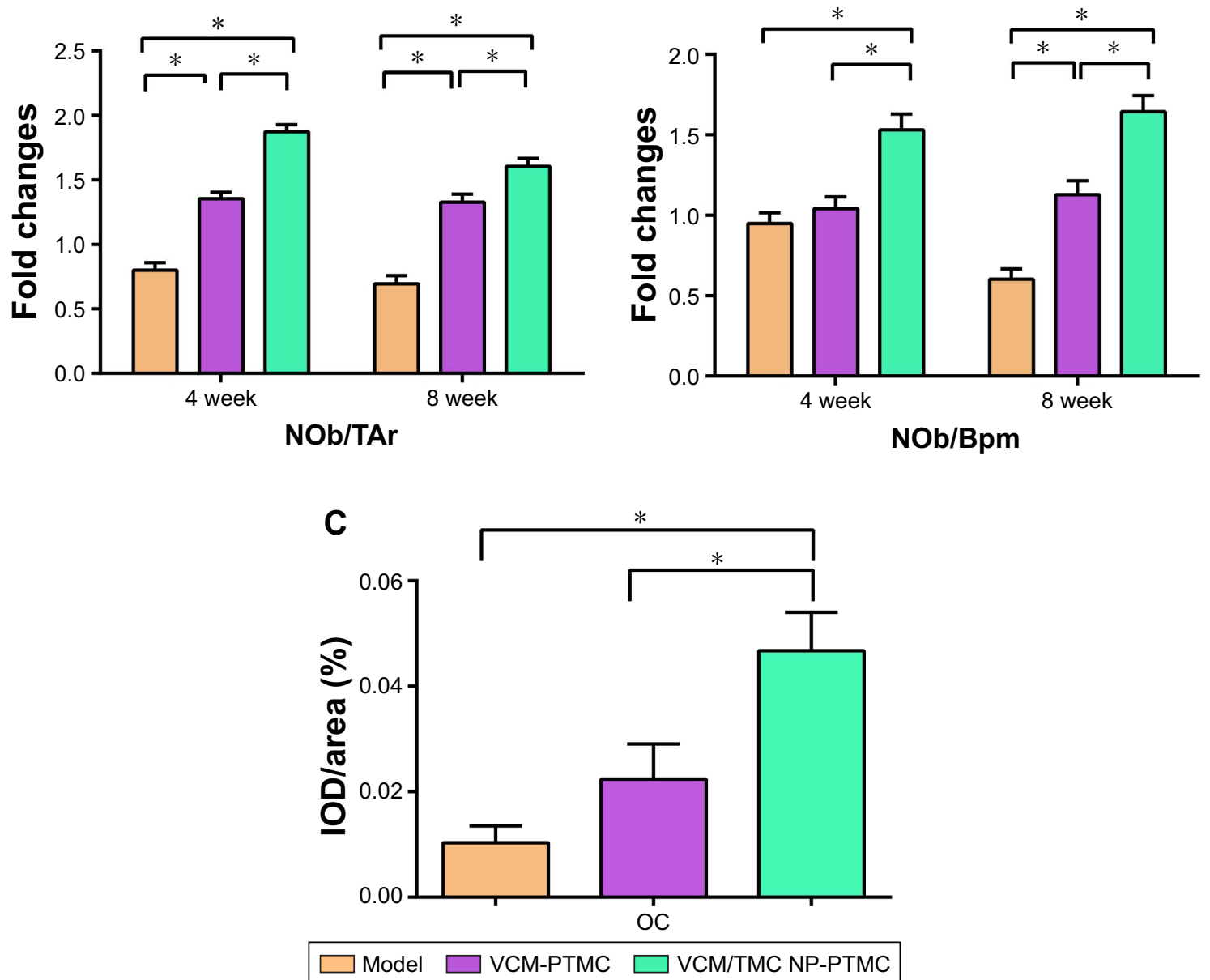

Figure 5 The histopathology of tibia and immunohistochemical analysis.

Notes: (A) Typical histopathology of the rabbit tibia. (B) The columns represent the scores of osteoblast number/trabecular bone area (NOb/TAr); osteoblast number/ bone perimeter (NOb/Bpm). (C) The columns represent the IOD/area rate of immunohistochemical staining for OC from six rabbits per group. Data are presented as the mean \pm SD. $* P<0.05$

Abbreviations: IOD, integrated optical density; NP, nanoparticle; OC, osteocalcin; PTMC, poly(trimethylene carbonate); SD, standard deviation; TMC, N-trimethyl chitosan; VCM, vancomycin. 
possesses more hydroxyl termini, it is liable to absorb water and is not as subject to enzymatic degradation. ${ }^{32,33}$ Overall, the release of VCM/TMC NP-PTMC may be better for the prolonged administration of antibiotics. ${ }^{34}$

Protein adsorption was enhanced in the VCM/TMC NPPTMC, mainly due to 1) the presence of NPs that increase the total surface area and binding sites for proteins or 2) an electrostatic interaction between the material surface and proteins. Protein adsorption further aids in cellular adhesion, infiltration and proliferation by the adsorption of adhesion molecules such as fibronectin and/or vitronectin. ${ }^{35,36}$

The adhesion and proliferation of ROBs could be influenced by varying the nanostructured surfaces. The fixed surface structure in the nanometer-size scale could be useful for designing novel scaffolds in tissue engineering applications. ${ }^{37}$ Therefore, it is reasonable to conclude that the VCM/TMC NP-PTMC are not only cytocompatible but also significantly effective on ROB proliferation to induce bone regeneration. PTMC is recognized as a cell-friendly material as well, with high levels of cell viability and cell adhesion being observed on the PTMC surface. ${ }^{38}$ As a result, VCM/TMC NP-PTMC can be useful nanocarriers for the intracellular delivery of various antibiotics.

The different antibacterial activities in vitro were due to the presence of VCM/TMC NPs in the VCM/TMC NPPTMC, possessing the slow biodegradation and sustained drug release characteristics of VCM/TMC NPs. Moreover, in our previous study, ${ }^{39}$ the synthesis mechanism and cell uptake evaluation proved that the VCM/TMC NPs with a positive zeta potential were responsible for the effective attachment to the negatively charged cell membrane of bacteria, which caused the leakage of proteinaceous and intracellular components, leading to bacterial rupture and death. In this study, the VCM/TMC NP-PTMC could improve delivery of watersoluble VCM across the cytomembrane, due to its positive surface charge, facilitating the movement of antibiotics across the ROB membrane. Therefore, antibiotic treatment of the infected osteoblasts indicated that VCM/TMC NP-PTMC inhibited intracellular $S$. aureus efficiently, which can explain the excellent antibiotic activity of VCM/TMC NP-PTMC in the chronic osteomyelitis rabbits.

In chronic osteomyelitis, although the necrotic tissue has been adequately debrided, a large bone defect is inevitable. As a result, the appropriate reconstruction of bone defect is needed. VCM-CaSO ${ }_{4}$ was used for local antibiotic treatment for many years in clinic; however, the overdosage of VCM administered locally caused the inhibition of osteoblast activity and proliferation. ${ }^{40}$ NPs have been shown to promote cell attachment and proliferation ability. ${ }^{41,42}$ Actually, the VCM/TMC NP-PTMC in our study exhibited a sustained and slow release that can eliminate the inhibition of high dose of VCM and possessed excellent antibiotic activity and bone repair capability. Meanwhile, the PTMC is best known as a surface-eroding polymer and is fully biocompatible, which appears to fully degrade in bone and assists in promoting bone regeneration. ${ }^{18,21}$ Generally, in the clinic, patients are commonly supplied therapy for at least 4 weeks. Therefore, VCM/TMC NP-PTMC could meet the requirements.

Although, the proliferative probability of ROBs and the antibacterial activities of VCM/TMC NPs and VCM/TMC NP-PTMC in vivo and in vitro were compared and evaluated in this article, the molecular mechanism of effect of VCM/ TMC NPs and VCM/TMC NP-PTMC on bone regeneration and bone repair has not been stated. The relevant studies will be performed in our further research.

\section{Conclusion}

This work has demonstrated that rationally designed VCM/ TMC NP-PTMC can be readily prepared using a composite method with a high drug-loading capacity and a steady, biodegradable, cytocompatible and antibacterial nature. An in vivo study showed that implantation of VCM/TMC NP-PTMC was an efficient method of antibiotic treatment in chronic osteomyelitis, and VCM/TMC NP-PTMC had excellent probability of promoting bone healing. These NPs thus can be a promising strategy to treat intracellular infections such as chronic osteomyelitis in the clinic.

\section{Acknowledgments}

This study was financially supported by the Zhejiang Provincal Natural Science Foundation (LY17H290002), the Science and Technology project of Zhejiang Province (2015F50018, 2016RCB003), the Science and Technology Innovation Team Project of Ningbo Science and Technology Bureau (2015C110027).

\section{Disclosure}

The authors report no conflicts of interest in this work.

\section{References}

1. Sugaya H, Mishima H, Aoto K, et al. Percutaneous autologous concentrated bone marrow grafting in the treatment for nonunion. Eur J Orthop Surg Traumatol. 2014;24(5):671-678.

2. Xie Z, Liu X, Jia W, Zhang C, Huang W, Wang J. Treatment of osteomyelitis and repair of bone defect by degradable bioactive borate glass releasing vancomycin. J Control Release. 2009;139(2):118-126.

3. Abed N, Couvreur P. Nanocarriers for antibiotics: a promising solution to treat intracellular bacterial infections. Int J Antimicrob Agents. 2014; 43(6):485-496. 
4. Liu J, Volk KJ, Lee MS, Pucci M, Handwerger S. Binding studies of vancomycin to the cytoplasmic peptidoglycan precursors by affinity capillary electrophoresis. J Anal Chem. 1994;66(14):2412-2416.

5. Li B, Brown KV, Wenke JC, Guelcher SA. Sustained release of vancomycin from polyurethane scaffolds inhibits infection of bone wounds in a rat femoral segmental defect model. J Control Release. 2010;145(3): 221-230.

6. Walter G, Kemmerer M, Kappler C, Hoffmann R. Treatment algorithms for chronic osteomyelitis. Dtsch Arztebl Int. 2012;109(14):257-264.

7. Henriksen K, Neutzsky-Wulff AV, Bonewald LF, Karsdal MA. Local communication on and within bone controls bone remodeling. Bone. 2009; 44(6):1026-1033.

8. Shou D, Dong Y, Shen LF, et al. Rapid quantification of tobramycin and vancomycin by UPLC-TQD and application to osteomyelitis patient samples. J Chromatogr Sci. 2014;52:501-507.

9. Shen LF, Dong Y, Zhang C, et al. Chronic osteomyelitis treatment: a clinical and pharmaco-kinetic study of vancomycin impregnated calcium sulphate. J Med Imag Health In. 2015;5:36-42.

10. Uskokovic V, Desai TA. Simultaneous bactericidal and osteogenic effect of nanoparticulate calcium phosphate powders loaded with clindamycin on osteoblasts infected with Staphylococcus aureus. Mater Sci Eng C. 2014;37:210-222.

11. Huh AJ, Kwon YJ. "Nanoantibiotics": a new paradigm for treating infectious diseases using nanomaterials in the antibiotics resistant era. J Control Release. 2011;156:128-145.

12. Katas H, Hussain Z, Ling TC. Chitosan nanoparticles as a percutaneous drug delivery system for hydrocortisone. J Nanomater. 2012;3: 2817-2827.

13. Guan M, Zhu QL, Liu Y, et al. Uptake and transport of a novel anticancer drug-delivery system: lactosyl-norcantharidin-associated $N$-trimethyl chitosan nanoparticles across intestinal Caco-2 cell monolayers. Int J Nanomed. 2012;7:1921-1930.

14. Wu F, Meng G, He J, Wu Y, Wu F, Gu ZW. Antibiotic-loaded chitosan hydrogel with superior dual functions: antibacterial efficacy and osteoblastic cell responses. ACS Appl Mater Interfaces. 2014;6(13): 10005-10013.

15. Zhao L, Zhu BY, Jia YH, Hou WJ, Sue C. Preparation of biocompatible carboxymethyl chitosan nanoparticles for delivery of antibiotic drug. Biomed Res Int. 2013;2013:485-496.

16. Vongchana P, Wutti-Ina Y, Sajomsang W, Gonil P, Kothan S, Linhardt RJ. N,N,N-Trimethyl chitosan nanoparticles for the delivery of monoclonal antibodies against hepatocellular carcinoma cells. Carbohydr Polym. 2011;85(1):215-220.

17. Xu JJ, Xu BH, Shou D, Xia XJ, Hu Y. Preparation and evaluation of vancomycin-loaded $N$-trimethyl chitosan nanoparticles. Polymers Basel. 2015; 7:1850-1870.

18. Bat E, van Kooten TG, Feijen J, Grijpma DW. Macrophage-mediated erosion of gamma irradiated poly(trimethylene carbonate) films Biomaterials. 2009;30(22):3652-3661.

19. Fukushima K. Poly(trimethylene carbonate)-based polymers engineered for biodegradable functional biomaterials. Biomater Sci. 2016; 4(1):9-24.

20. Zhang Z, Kuijer R, Bulstra SK, Grijpma DW, Feijen J. The in vivo and in vitro degradation behavior of poly(trimethylene carbonate) Biomaterials. 2006;27(9):1741-1748.

21. Van Leeuwen AC, van Kooten TG, Grijpma DW, Bos RRM. In vivo behaviour of a biodegradable poly(trimethylene carbonate) barrier membrane: a histological study in rats. J Mater Sci. 2012;23(8):1951-1959.

22. Klui OS, van der Mei HC, Busscher HJ, Neut D. A surface-eroding antibiotic delivery system based on poly-(trimethylene carbonate). Biomaterials. 2009;30(27):4738-4742.

23. Zhang Y, Wu RJ, Hu Y, et al. A dynamic eluting mode to investigate drug release of antibiotic-impregnated calcium sulfate delivery system for the clinical purpose. J Mech Med Biol. 2015;15:1550012.
24. Bakker A, Klein-Nulend J. Osteoblast isolation from murine calvariae and long bones. Methods Mol Med. 2003;80:19-28.

25. Uskoković V, Desai TA. Simultaneous bactericidal and osteogenic effect of nanoparticulate calcium phosphate powders loaded with clindamycin on osteoblasts infected with Staphylococcus aureus. Mater Sci Eng C Mater Biol Appl. 2014;37:210-222.

26. Shalumon KT, Sowmya S, Sathish D, Chennazhi KP, Nair SV, Jayakumar R. Effect of incorporation of nanoscale bioactive glass and hydroxyapatite in PCL/chitosan nanofibers for bone and periodontal tissue engineering. J Biomed Nanotechnol. 2013;9(3):430-440.

27. Srinivasan S, Kumar S, Nair SV, Nair SV, Chennazhi KP, Rangasamy J. Antibacterial and bioactive $\alpha$ - and $\beta$-chitin hydrogel/nanobioactive glass ceramic/nano silver composite scaffolds for periodontal regeneration. J Biomed Nanotechnol. 2013;9:1803-1816.

28. Norden CW, Myerowitz RL, Keleti E. Experimental osteomyelitis due to Staphylococcus aureus or Pseudomonas aeruginosa: a radiographicpathological correlative analysis. Br J Exp Pathol. 1980;61(4): 451-460.

29. Shou D, Zhang Y, Shen LF, et al. Flavonoids of Herba Epimedii enhances bone repair in a rabbit model of chronic osteomyelitis during post-infection treatment and stimulates osteoblast proliferation in vitro. Pytother Res. 2016.

30. Wei Y, Xu L, Tao YX, Yao C, Xue HG, Kong Y. Electrosorption of lead ions by nitrogen-doped graphene aerogels via one-pot hydrothermal route. Ind Eng Chem Res. 2016;55:1912-1920.

31. Yang J, Tian W, Li Q, Li Y, Cao A. Novel biodegradable aliphatic poly(butylene succinate-co-cyclic carbonate)s bearing functionalizable carbonate building blocks: II. Enzymatic biodegradation and in vitro biocompatibility assay. Biomacromolecules. 2004;5(6):2258-2268.

32. Vyner MC, Li A, Amsden BG. The effect of poly(trimethylene carbonate) molecular weight on macrophage behavior and enzyme adsorption and conformation. Biomaterials. 2014;35(33):9041-9048.

33. Fukushima K, Tsai MY, Ota T, et al. Evaluation of the hemocompatibility of hydrated biodegradable aliphatic carbonyl polymers with a subtle difference in the backbone structure based on the intermediate water concept and surface hydration. Polym J. 2015;47:469-473.

34. Rao N, Lipsky BA. Optimising antimicrobial therapy in diabetic foot infections. Drugs. 2007;67(2):195-214.

35. Srinivasan S, Jayasree R, Chennazhi KP, Nair SV, Jayakumar R. Biocompatiblealginate/nano bioactive glass ceramic composite scaffolds for periodontal tissue regeneration. Carbohydr Polym. 2012;87: 274-283.

36. Peter M, Kumar S, Sathy BN, Rangasamy J. Development of novel $\alpha$-chitin/nanobioactive glass ceramic composite scaffolds for tissue engineering applications. Carbohydr Polym. 2009;78:926-931.

37. Wardwell PR, Forstner MB, Bader RA. Investigation of the cytokine response to NF- $\kappa \mathrm{B}$ decoy oligonucleotide coated polysaccharide based nanoparticles in rheumatoid arthritis in vitro models. Arthritis Res Ther. 2015;17:310.

38. Pirhonen E, Törmälä P. Coating of bioactive glass 13-93 fibres with biomedical polymers. J Mater Sci. 2006;41:2031-2036.

39. Arpornwichanop T, Polpanich D, Thiramanas R, Suteewong T, Tangboriboonrat P. PMMA-N,N,N-trimethyl chitosan nanoparticles for fabrication of antibacterial natural rubber latex gloves. Carbohydr Polym. 2014;109:1-6.

40. Mendoza MC, Sonn KA, Kannan AS, et al. The effect of vancomycin powder on bone healing in a rat spinal rhBMP-2 model. J Neurosurg Spine. 2016;25(2):147-153.

41. Keijzer C, Slutter B, van der Zee R, Jiskoot W, van Eden W, Broere F. PLGA, PLGA-TMC and TMC-TPP nanoparticles differentially modulate the outcome of nasal vaccination by inducing tolerance or enhancing humoral immunity. PLoS One. 2011;6(11):e26684.

42. Zhang N, Bader RA. Synthesis and characterization of polysialic acid- $N$-trimethyl chitosan nanoparticles for drug delivery. Nano LIFE. 2012;2(3):1241003. 


\section{Publish your work in this journal}

The International Journal of Nanomedicine is an international, peerreviewed journal focusing on the application of nanotechnology in diagnostics, therapeutics, and drug delivery systems throughout the biomedical field. This journal is indexed on PubMed Central, MedLine, CAS, SciSearch $\AA$, Current Contents $\AA /$ Clinical Medicine,

Journal Citation Reports/Science Edition, EMBase, Scopus and the Elsevier Bibliographic databases. The manuscript management system is completely online and includes a very quick and fair peer-review system, which is all easy to use. Visit http://www.dovepress.com/ testimonials.php to read real quotes from published authors.

Submit your manuscript here: http://www.dovepress.com/international-journal-of-nanomedicine-journal 